\title{
DeepCMB: Saliency for Image-to-Image Regression
}

\section{J. Caldeira ${ }^{1 *}$, W. L. K. Wu², B. Nord ${ }^{123}$}

${ }^{1}$ Fermilab, ${ }^{2}$ Kavli Institute for Cosmological Physics, ${ }^{3}$ UChicago Astronomy and Astrophysics

*Corresponding author: caldeira@fnal.gov; A Deep Skies Lab Joint (deepskieslab.com); arXiv:1810.01483 + upcoming

FERMILAB-POSTER-19-128-AE-SCD

\section{How can we reconstruct the CMB lensing potential at the noise levels of future experiments?}

Problem

statement:

recovering the

lensing signal from

the measured

lensed maps.
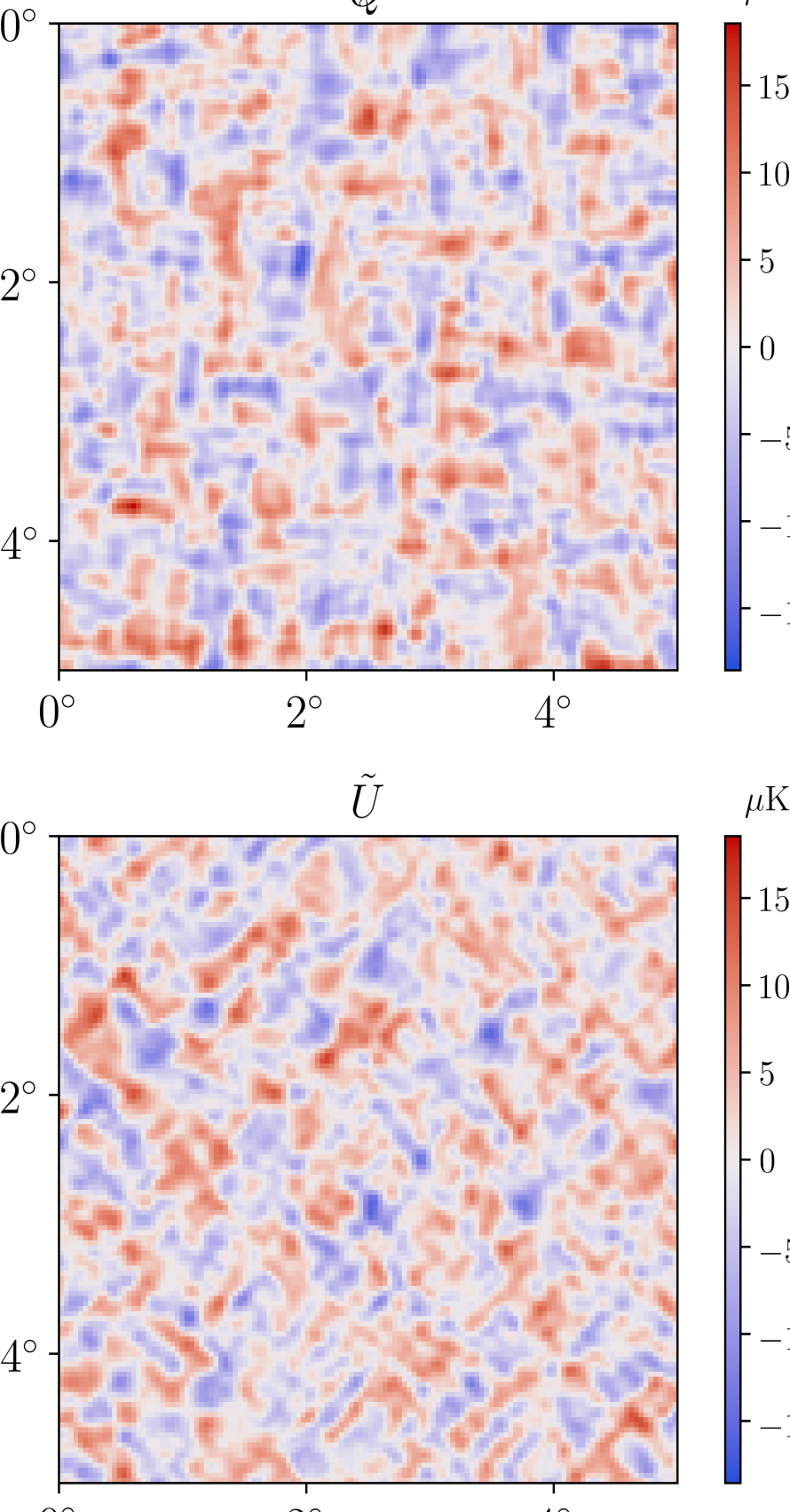
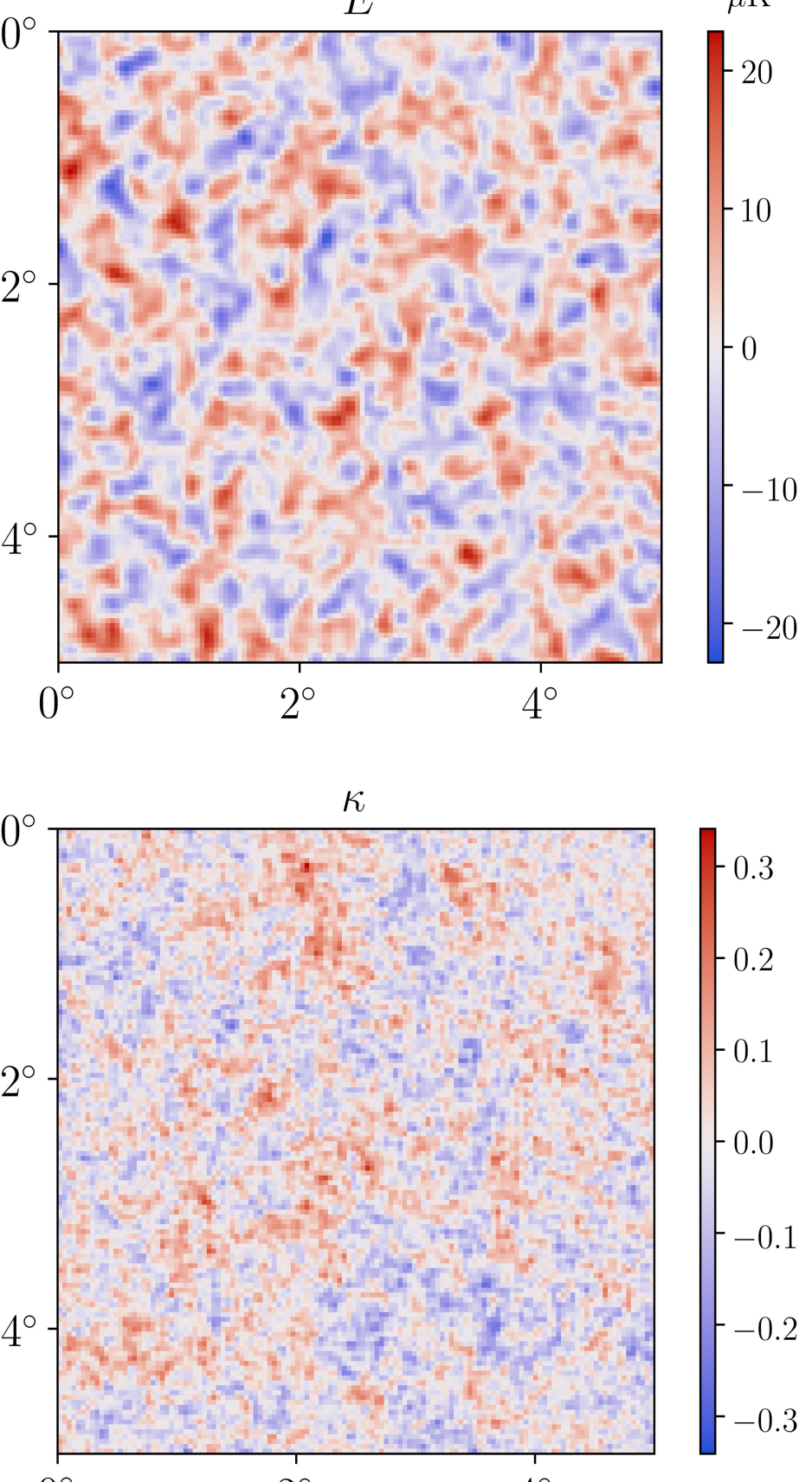

Current methods will be suboptimal at noise levels of next-generation experiments. Maximum likelihood methods will be required. Can neural networks help? Yes.

\section{The network architecture: first encode a useful representation of the inputs, then process it to outputs.}

We use a Residual U-Net (ResUNet). Each orange box includes a dropout layer, a $5 \times 5$ convolution, application of a SELU activation function, and batch normalization. The network is trained on $5^{\circ} \times 5^{\circ}$ patches of sky generated based on $\Lambda$ CDM cosmology.

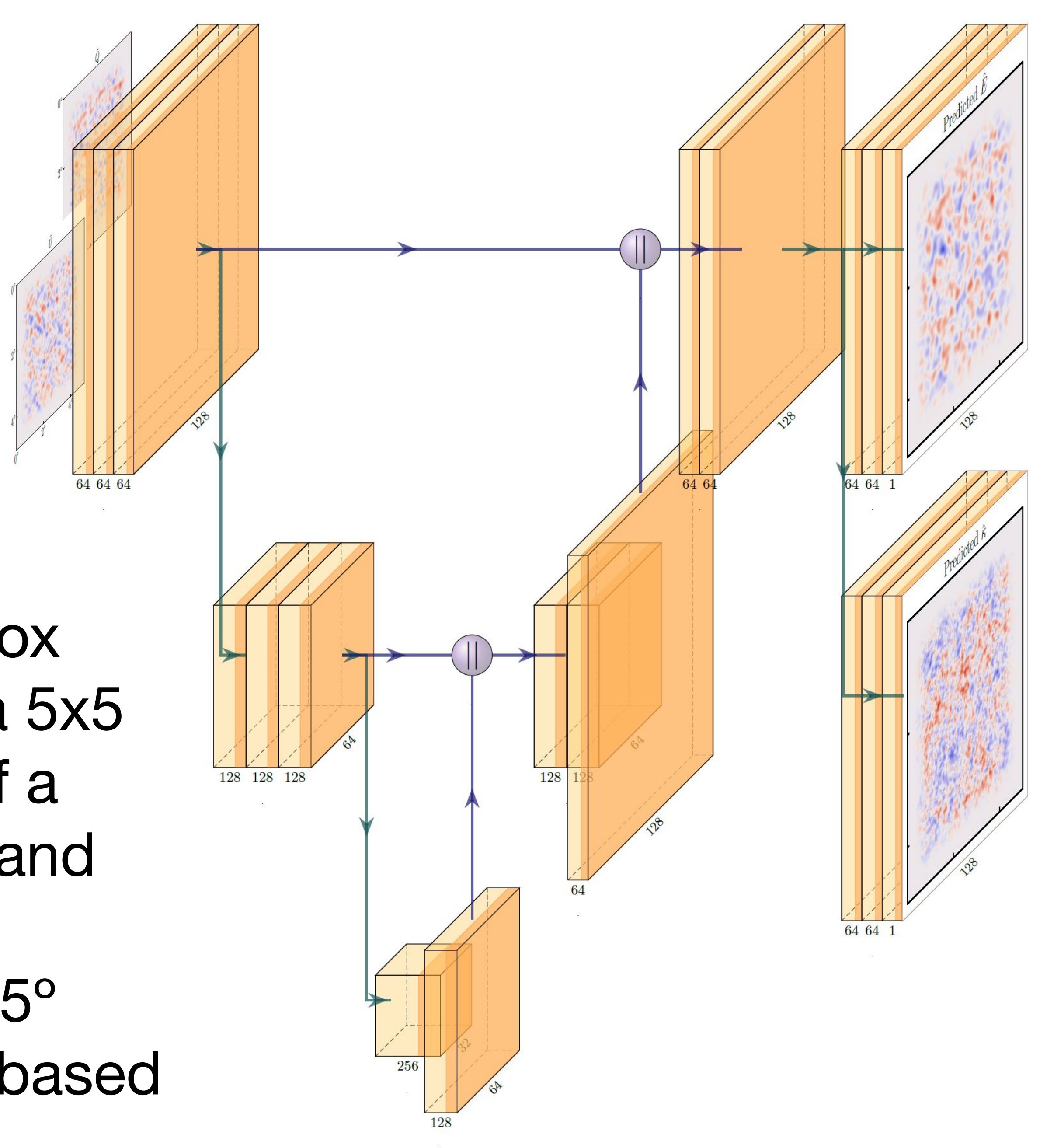

Results: Recovery of $E$ and convergence for $0,1,5 \mu \mathrm{K}$-arcmin noise

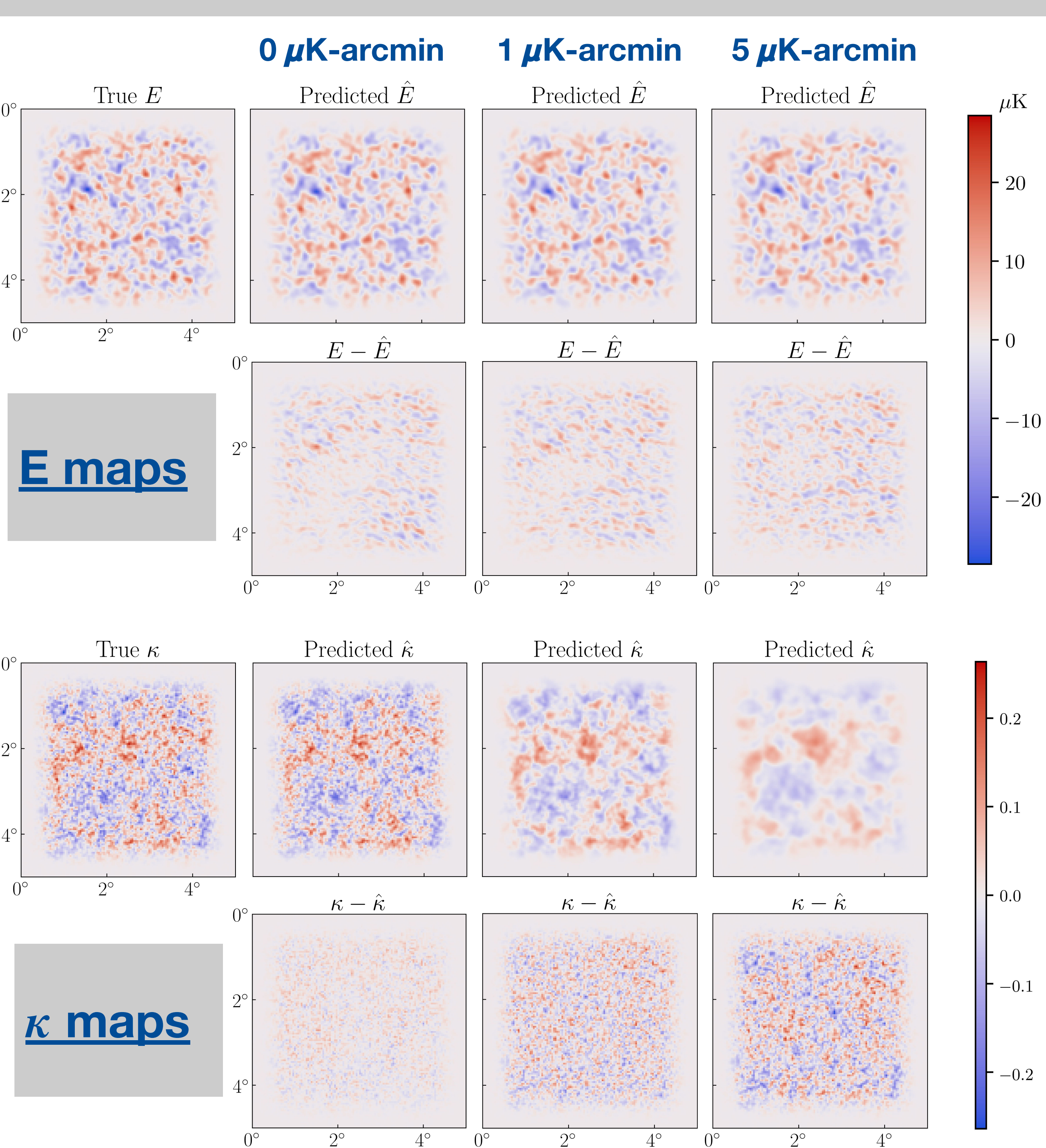

For noiseless

maps, $\kappa$ recovery is better than $E$ recovery. However, adding noise affects $\kappa$ recovery much more, and by $5 \mu \mathrm{K}$-arcmin only the large-scale structure remains.

But how can we more closely examine the physics learned?

\section{Saliency maps}

What is saliency?

Writing the network map as $f(Q, U)$, saliency evaluates $\left|f^{\prime}(Q, U)\right|$ at points corresponding to a specific input image. This allows us to infer which pixels matter most for a prediction. All results below are averaged over 100 images in the validation set.

\section{$\underline{\text { Results for all pixels }}$}

Maps should be uniform. However, for ResUNet (left), they are not! The effect comes from strides downsampling, and disappears when a flat network is used instead (right).
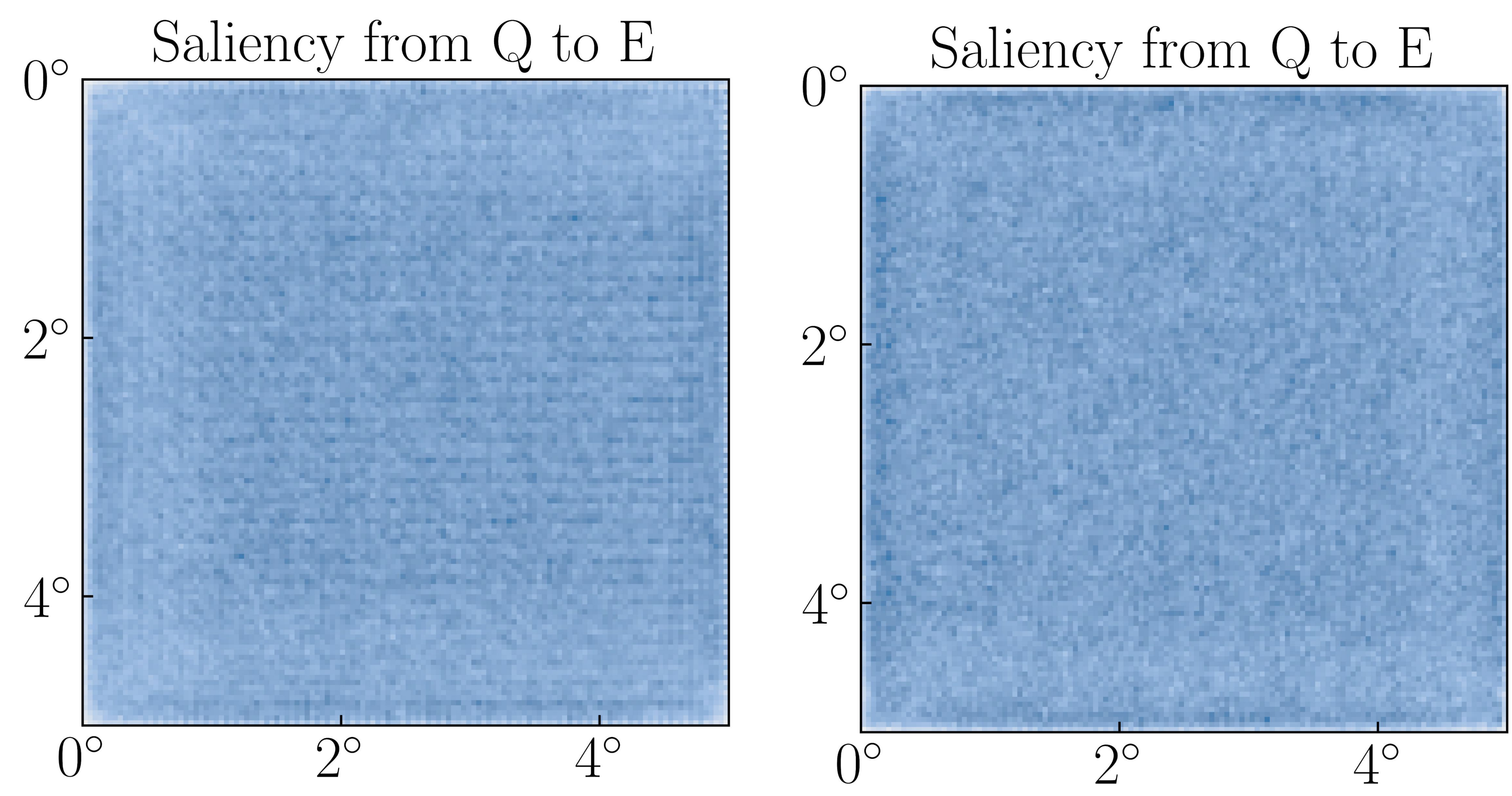

Results for single pixels

We can see that the network learns $Q$ to $k$ is local, while $Q$ to $E$ is non-local (local in Fourier space).
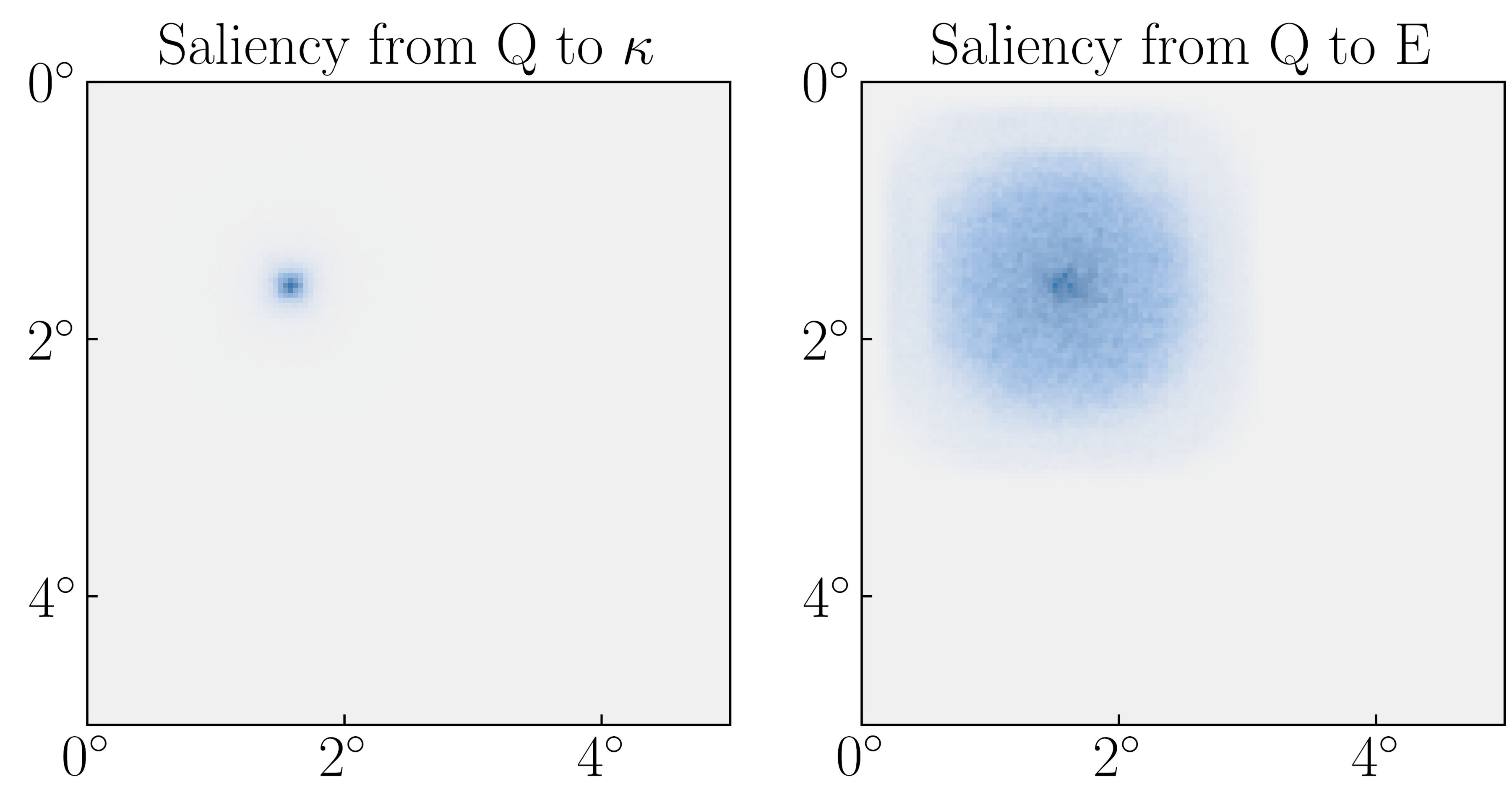

Saliency also helps us understand how deep the network should be to reflect the right physics!

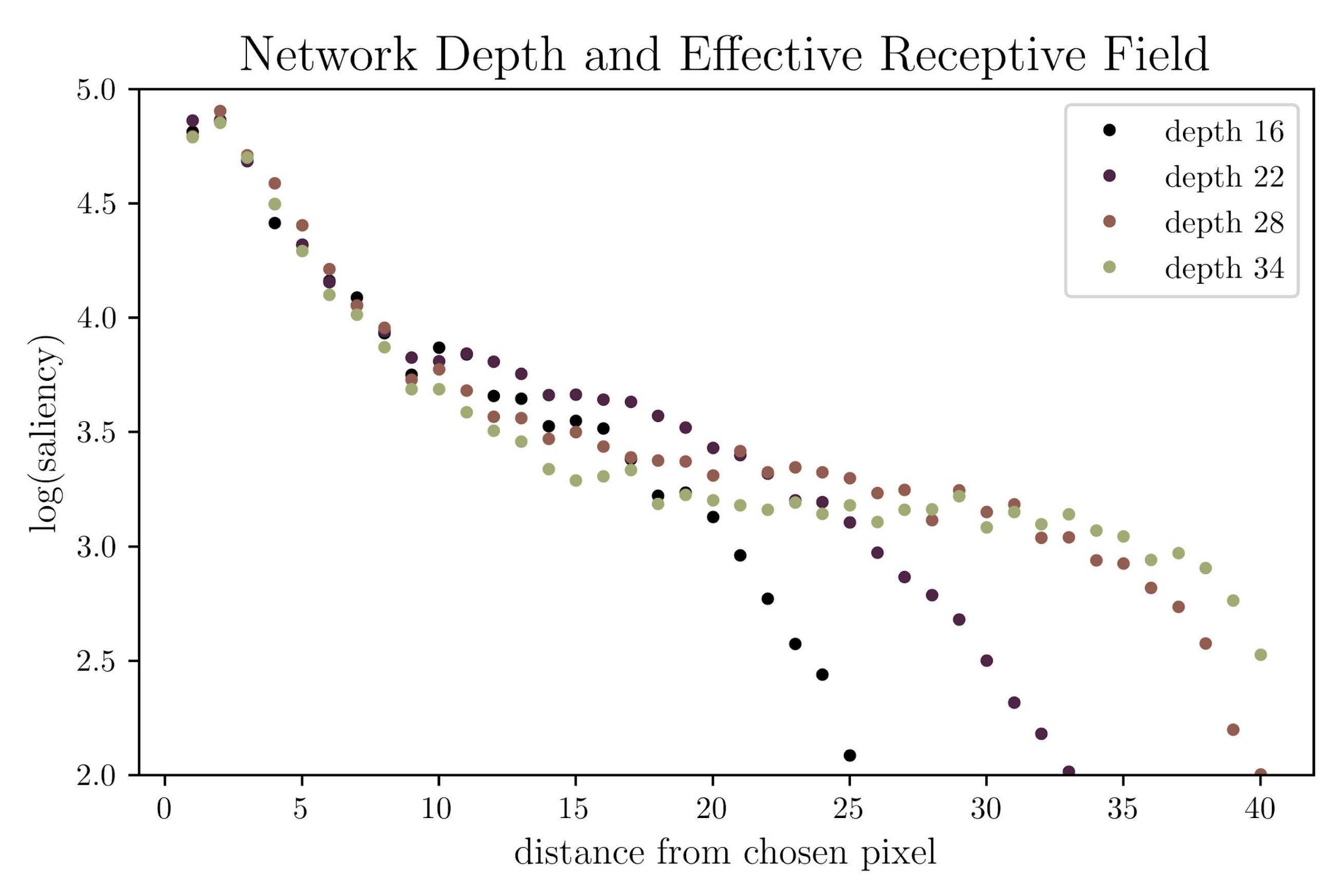

This manuscript has been authored by Fermi Research Alliance, LLC under Contract No. DE-AC02-07CH11359 with the U.S. Department of Energy, Office of Science, Office of High Energy Physics. 